\title{
Oxidative Stress Therapy for Cancer Using Glycolysis Inhibitors: Towards Improving Therapeutic Outcomes
}

\author{
Salah Mohamed EI Sayed ${ }^{1,2, *}$ \\ ${ }^{1}$ Department of Clinical Biochemistry and Molecular Medicine, Taibah College of Medicine, Taibah University, \\ Al-Madinah Al-Munawwarah, Saudi Arabia \\ ${ }^{2}$ Department of Medical Biochemistry, Sohag Faculty of Medicine, Sohag University, Egypt \\ *Corresponding author: salahfazara@yahoo.com, drsalahpediatr@yahoo.com
}

\begin{abstract}
Cancer cure is still a big challenge despite intensive conventional treatment. Unfortunately, many unsolved problems e.g. metastasis and drug resistance still exist. Better understanding of cancer biology to identify important differences between cancer cells and normal cells seems vital to improve cancer treatment. Tumors depend on glycolysis for energy production, exhibit Warburg effect, establish aggressive microenvironment, have low antioxidant systems and are under high steady-state ROS conditions. Normal cells differ in all of that. Lactate produced through Warburg effect maintains the high steady-state ROS condition in cancer cells and helps cancer cells to metastasize and establish their hostile microenvironment. Cancer cells seem sensitive to oxidative stress therapy using ROS generating chemotherapy e.g. 3-bromopyruvate (3BP). 3BP is a powerful antiglycolytic that may be more promising as a potent anticancer if it is conjugated with polyethylene glycol (PEG). Targeting glycolysis in cancer cells seems promising in decreasing their survival and metastasis. Glycolysis double inhibition by combination of multiple antiglycolytics e.g. 3BP with citrate was synergistic in cancer treatment. Being analog to pyruvate and lactate, 3BP antagonized Warburg effect, antagonized effects of pyruvate and lactate, improved sensitivity of chemoresistant cancer cells and targeted cancer cell survival, migration and metastasis. In this review, I discuss 3BP-induced oxidative stress and ATP depletion as a promising treatment modality for cancer.
\end{abstract}

Keywords: 3-bromopyruvate, EPR effect, PEG formulation, glycolysis, citrate

Cite This Article: Salah Mohamed El Sayed, "Oxidative Stress Therapy for Cancer Using Glycolysis Inhibitors: Towards Improving Therapeutic Outcomes." American Journal of Medical and Biological Research, vol. 6, no. 1 (2018): 11-15. doi: 10.12691/ajmbr-6-1-3.

\section{Introduction}

Better understanding of cancer biology to identify important differences between cancer cells and normal cells seems vital to improve cancer treatment. Tumors depend on glycolysis for energy production, exhibit Warburg effect, establish aggressive microenvironment, have low antioxidant systems and are under high steadystate ROS conditions. Normal cells differ in all of that. Lactate produced through Warburg effect maintains the high steady-state ROS condition in cancer cells and helps cancer cells to metastasize and establish their hostile microenvironment. Cancer cells seem sensitive to oxidative stress therapy using ROS generating chemotherapy e.g. 3-bromopyruvate (3BP) [1].

Oxidative stress therapy is a promising therapy to cancer cells provided that normal cells do not suffer badly. Reactive oxygen species (ROS)-mediated cytotoxicity to cancer cells may be utilized as a methodology for treating malignant tumors via inducing oxidative stress. Oxidative stress therapy aims at overproduction of ROS exclusively in cancer cells to avoid harming normal tissues. Long-standing endogenous oxidative stress in malignant cells may force cancer cells to develop a strong antioxidative system which protects malignant cells from oxidative stress $[2,3,4]$. Those antioxidant mechanisms developed by cancer cells protect them against ROS, enhance chemoresistance, facilitate metastasis and inhibit apoptosis in cancer cells [5,6,7]. Moreover, ROS can induce activity of antioxidants in cancer cells e.g. NO is well-known to be a potent inducer of heme oxygenase-1 (HO-1), which functions as an antioxidant enzyme to protect tumor cells against oxidative stress $[8,9,10]$. HO-1 was reported to be up-regulated in many cancer types e.g. prostate [11], brain [12] and renal cancers [13].

\section{EPR Effect and Strategies for Oxidative Therapy}

Hydrogen peroxide $\left(\mathrm{H}_{2} \mathrm{O}_{2}\right)$ is cytotoxic to cancer cells in a dose-dependent manner. Production of $\mathrm{H}_{2} \mathrm{O}_{2}$ is shared by many currently used chemotherapeutics e.g. cisplatin [14]. Oxidative stress therapy was introduced to utilize ROS generating properties exerted by specific enzymes, which do so upon acting on their substrates e.g. glucose oxidase (GO) and xanthine oxidase (XO). XO-polymer conjugates induced powerful generation of free radicals in tumor tissues [15]. GO was not preferable as a candidate for oxidative stress therapy as its substrate (D-glucose) is 
enormously available in the body which makes it difficult to control ROS production.

A practical therapeutic problem soon appeared which affected the power of $3 \mathrm{BP}$ as an excellent tool for oxidative therapy. That was related to the short in vivo half-life of 3BP which shortens the oxidative stress effect induced by 3BP. The small molecular size of 3BP (166.96 $\mathrm{g} / \mathrm{mol}$ ) does not allow 3BP to accumulate in tumor tissues (enhanced permeability and retention effect, EPR effect) as MW of 3BP is relatively small and may allow loss of infused 3BP through renal clearance [16-17]. EPR effect is a molecular size-dependent phenomenon that works with a large molecular size of molecules (40 kDa) [18]. Solving that problem may occur through conjugating 3BP to relatively large-sized molecules (Figure 1).

Biocompatible macromolecules e.g. polyethylene glycol (PEG) can accumulate and persist for a long time in the blood vessels of solid tumors because of the high porosity of tumor vasculature together with the impaired lymphatic clearance system [17]. The EPR effect of macromolecules and lipids in solid tumors made a basis for increasing the molecular weight of therapeutic proteins and drugs for proper prolongation of their effects in vivo. Based on the EPR effect, 3BP conjugated to PEG (PEG-3BP) may be significantly stronger as a tool for inducing oxidative stress therapy than crude 3BP [17]. 3BP PEGylation increases the molecular weight and half-life of 3BP. PEG-3BP can accumulate in tumor tissues selectively where high 3BP plasma level can remain for a long period (Figure 1). Targeted delivery of PEG-3BP can be achieved for antitumor therapy via enzymatic generation of hydrogen peroxide [17].
Strategies for ROS-mediated treatment of cancer aim at exposing cancer cells directly to ROS generating agents, inhibition of protection that may be conferred by antioxidants, reducing the cellular-oxidant buffering capacity or a combination of all of that. There is a long list of the agents that work through oxidative stress pathways e.g. arsenic trioxide, anthracyclines, bleomycin, bortezomib and cisplatin. All work through ROS generation, while ascorbate and diethylmaleate work through depletion of antioxidants (GSH depletion). Mercaptosuccinic acid and ethacrynic acid act as inhibitors of antioxidant enzymes [18].

Targeting critical antioxidant enzymes that are upregulated in tumors e.g. HO-1 aimed at suppressing the antioxidant systems in many tumors. These antioxidative systems are important for tumor cells to defend themselves against the oxidative stress induced by ROS and reactive nitrogen species (RNS). Intra-arterial injection of zinc protoporphyrin IX ( $\mathrm{ZnPP})$, an inhibitor of HO-1, to solid tumors suppressed xenograft tumor growth [19]. Conjugation of PEG with $\mathrm{ZnPP}$ was reported later in which PEG-ZnPP was characterized by high water-solubility. PEG-Zn PP formed multimolecular associations with molecules larger than $70 \mathrm{kDa}$ in aqueous media which improved tumor-targeting efficiency [20]. Combination of oxidative stress therapy and PEG-ZnPP potentiated the chemotherapeutic response of tumor cells by enhancing the oxidative stress effects induced by oxidative stress therapy and minimizing the antioxidant effects induced by HO-1 [21]. This carries benefits and advantages of EPR effect for potentiating oxidative stress therapy effect and lowering HO-1 effect.

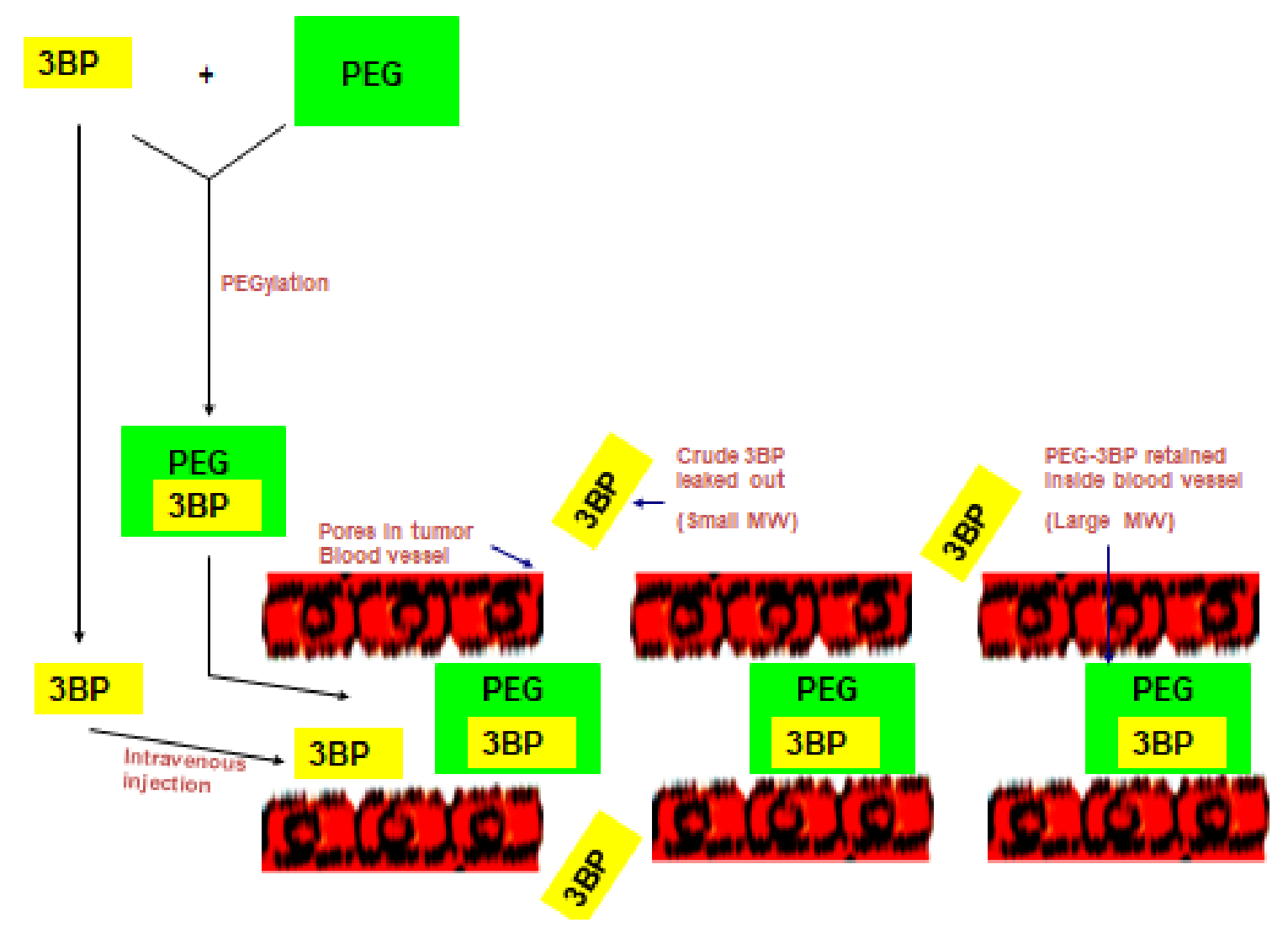

Figure 1. Enhanced permeability and retention (EPR) effect can be applied to 3BP treatment. Crude 3BP is low in molecular weight and may leak through the tumor vascular pores. Conjugation of 3BP with polyethylene glycol (PEG) helps retention of 3BP intravascularly to maintain a prolonged effect of 3BP-induced ATP depletion and oxidative stress therapy 


\section{Relationship between Oxidative Stress Therapy and Glycolysis Inhibition}

Oxidative stress is closely related to ATP depletion i.e. oxidative stress causes significant dose-dependent ATP depletion [22] causing cancer cells apoptosis e.g. 3BP caused activation of procaspase-3 [23,24].

\section{C6 Glioma as a Model for GBM}

Gliomas especially the aggressive type glioblastoma multiforme (GBM) are among the tumors which resist current therapeutic modalities. Post-operative survival in GBM is still dismal despite aggressive conventional therapy due to recurrence of GBM. Interestingly, temozolomide (TMZ), one of the current chemotherapeutics for GBM exerts its effects through generation of $\mathrm{H}_{2} \mathrm{O}_{2}$ [25]. Oxidative stress therapy significantly decreased viability and proliferation power of C6 glioma cells in a dose- and time-dependent manner. Oxidative stress therapy also significantly decreased the clonogenic power and anchorage-independent growth power of gliomas in a dose-dependent manner which confirms the power of oxidative stress therapy as a suggested anticancer modality. Both clonogenic power and anchorage-independent growth power reflect the power of a single cancer cell to start a rapid continuous proliferation to form a colony in the former and to grow without being attached to a substratum in the latter.

Taking GBM as an example, current treatment modalities for GBM are still not effective e.g. surgical removal of tumors followed by radiotherapy and chemotherapy (multimodal interventions) did not improve the dismal prognosis of patients where the mean patients' survival is about 6 to 12 months after the time of diagnosis [26]. Much better experimental results in GBM were obtained through significantly decreasing lactate extrusion which led to decreased invasiveness of GBM cells and increased apoptosis, while the survival of tumor-bearing experimental animals was significantly prolonged. Interestingly, inhibition of lactate efflux using cinnamic acid derivatives sensitized glioma cells to radiotherapy through reducing radio-protective metabolites e.g. reduced glutathione and taurine [27].

Interestingly, it was reported that monotherapy using 3BP efficiently killed the chemoresistant human GBM cell line U373MG in a dose- and time-dependent manner [23]. Moreover, 3BP induced necrosis and apoptosis of C6 glioma cells [22].

\section{Glycolysis Double and Triple Inhibition}

3BP acts as a functional antagonist to lactate regarding its effects on viability of C6 glioma cells upon which both $3 \mathrm{BP}$ and lactate exerted antagonistic effects. Lactate protected against 3BP-induced C6 glioma cell death which was overcome upon increasing the dose of 3BP [23]. Moreover, 3BP was reported to compete with lactate for MCT [28]. Lactate protected energetics of C6 glioma cells against 3BP-induced ATP depletion. Lactate-induced protection of C6 glioma energetics was overcome upon treatment with higher doses of 3BP. To elucidate the role of lactate on immunity against cancer, pretreatment of tumor spheroids with an inhibitor of lactate production resulted in an increased cytokine production [29].

Serial doses of 3BP exerted a synergistic effect with citrate in decreasing viability, migratory power and ATP levels in C6 glioma cells. Interestingly, citrate had a strong synergistic effect with serial doses of $3 \mathrm{BP}$ (glycolysis double inhibition). Low dose citrate synergized serial doses of 3BP and decreased maximally the survival of C6 glioma cells. Migratory power of C6 glioma cells was significantly suppressed when citrate was combined with 3BP. This may be regarded as a deenergizing effect that caused a stoppage or at least a delay in the migratory power of cancer cells, which reflects the inhibition exerted by glycolysis double inhibition against the migratory power of cancer cells. This may be further explained in light of the maximal depletion of ATP levels upon using glycolysis double inhibition [23].

Studying drug-drug interaction seems mandatory before selecting the best drug combinations. Regarding sodium fluoride (NaF) (inhibitor of enolase), it was recently reported to induce a dose-dependent decrease in viability of $\mathrm{C} 6$ glioma in millimolar range [23]. NaF is toxic at high doses and was reported to be used as a pesticide [30]. Fluoride is a component of tooth paste that has a bactericidal effect [31]. Low dose of $\mathrm{NaF}$ was reported to be used with antiglycolytic combination in the treatment of a panel of human cancers [32]. Noteworthy, NaF (3 $\mathrm{mM}$ ) combination was significant in inducing C6 glioma cell death with citrate $(3 \mathrm{mM})$ at low doses of both. $\mathrm{NaF}$ $(3 \mathrm{mM})$ combination with $3 \mathrm{BP}(15 \mu \mathrm{M})$ was stronger than either alone but evident antagonism occurred with serially increased doses of 3BP [23]. Glycolysis triple inhibition through combining low effective concentrations of three glycolysis inhibitors (3BP, citrate and $\mathrm{NaF}$ ) caused maximal C6 glioma cell death. Further studying of glycolysis triple inhibition seems important by testing effects of a sequential treatment in case of drug antagonism [23]. Sequential administration of chemotherapeutics may decrease the antagonistic cytotoxicity faced at simultaneous drug administration e.g. in treating slowly growing human colon carcinoma HT-29 cells using camptothecin and etoposide, both were antagonistic at simultaneous administration. However, this antagonism significantly decreased when both drugs were used sequentially [33].

Based on structural similarity and common transportation through MCT, both pyruvate and lactate antagonized the effects of 3BP significantly in a dose-dependent manner. Lactate and pyruvate did not protect against C6 glioma cell death induced by $\mathrm{NaF}$ or citrate [23].

\section{Glycolysis Inhibitors in Treating Human Cancer}

Interestingly, an early study (done by Black et al.) reported the use of a combination of glycolytic inhibitors in treating many human patients suffering from a panel of different types of malignancies e.g. hematological cancer and digestive tract cancer. The cancer cases studied were advanced stages of cancer in which patients were surgically inoperable and could not tolerate radiation therapy. In almost all cases, the diagnosis of cancer was confirmed by biopsy. Drug treatment used was in the form 
of $\mathrm{NaF}$, iodoacetic acid (GAPDH inhibitor), malonic acid, and sodium azide. Significant objective and subjective benefits were observed. Responsive group was in the hematological cancer group e.g. Hodgkin's lymphoma, lymphosarcoma and acute myelogenous leukemia. Treatment was tolerated in $75 \%$ of cases. Side effects occurred in the other $25 \%$ of cases and were mostly in the form of nausea and vomiting [32].

Although this study was pioneering in the treatment of malignant tumors through targeting glycolysis in end-stage cancer patients, it may be useful to appreciate that early fruitful effort and mention some comments on it as a step towards improving this kind of future trials. Firstly, the authors did not report in this study any data about drug-drug interactions that may occur among the utilized antiglycolytic drugs. It seems necessary to do a prior in vitro testing to select the best synergistic combinations and to avoid the antagonistic combinations. Also, authors used $\mathrm{NaF}(80 \mathrm{mg})$ that is toxic in high doses [30], which may explain the nausea and diarrhoea. $\mathrm{NaF}$ interaction with tested agents was not reported. Secondly, the targeted enzymes in that study did not include the key glycolytic enzymes e.g. Hexokinase II, phosphofructokinase and pyruvate kinase. Thirdly, the non-responsive group included digestive and genital cancer groups in which the 4 tested agents were not effective. 3BP that was recently introduced as an anticancer agent [34] and was reported to be effective in treating digestive cancer both for in vitro and in vivo studies e.g. 3BP was powerful in advanced HCC [35], colon cancer [36] and pancreatic cancer [37]. Similar future studies utilizing $3 \mathrm{BP}$ are expected to improve the treatment response in the non-responsive digestive cancer group. Fourthly, sodium azide is not a glycolytic inhibitor but is an inhibitor of oxidative phosphorylation. Sodium azide was reported to cause an irreversible loss of cytochrome c oxidase activity via holoenzyme dissociation [38]. Also, malonic acid is an inhibitor of succinate dehydrogenase (an enzyme of Krebs cycle) [39]. Both oxidative phosphorylation and Krebs cycle are mitochondrial energy generating pathways that are less critical than glycolysis inhibition in cancer cells. This may explain the lack of response in some treatment groups.

\section{Conclusion}

3BP seems to be promising as a general anticancer agent for future treatment of cancer disease. 3BP-induced oxidative stress therapy and ATP depletion seems safe and less toxic towards normal cells. We hope that oxidative stress therapy can be a new promising line of treatment for cancer patients.

\section{Conflict of Interest}

The author declares that there is no conflict of interest.

\section{References}

[1] Baghdadi HH. Targeting Cancer Cells using 3-bromopyruvate for Selective Cancer Treatment. Saudi J Med Med Sci 2017; 5: 9-19.
[2] Pervaiz, S., Clement, M. V. (2004). Tumor intracellular redox status and drug resistance - serendipity or a causal relationship? Current Pharmacological Design 10(16), 1969-1977.

[3] Tiligada, E. Chemotherapy: induction of stress responses. (2006). Endocrine-related cancer, 13 Suppl. 1), S115-S124.

[4] Sullivan, R., Graham, C. H. (2008). Chemosensitization of cancer by nitric oxide. Current Pharmaceutical Design, 14, 1113-1123.

[5] Schneider, B. L., Kulesz-Martin, M. (2004). Destructive cycles: the role of genomic instability and adaptation in carcinogenesis. Carcinogenesis, 25, 2033-2044.

[6] Martinez-Sanchez, G., Giuliani, A. (2007). Cellular redox status regulates hypoxia inducible factor-1 activity. Role in tumor development. Journal of Experimental \& Clinical Cancer Research, 26, 39-50.

[7] Chen, E.I., Hewel, J., Krueger, J.S., Tiraby, C., Weber, M.R., Kralli, A., et al. (2007). Adaptation of energy metabolism in breast cancer brain metastases. Cancer Research, 67(4), 1472-1486.

[8] Hara, E., Takahashi, K., Takeda, K., Nakayama, M., Yoshizawa, M., Fujita, H. et al. (1999). Induction of heme oxygenase-1 as a response in sensing the signals evoked by distinct nitric oxide donors. Biochemical Pharmacology, 58, 227-236.

[9] Hartsfield, S.L, Alam, J., Cook, J.L., Choi, AM. (1997). Regulation of heme oxygenase-1 gene expression in vascular smooth muscle cells by nitric oxide. The American Journal of Physiology, 273, 980-988.

[10] Doi, K., Akaike, T., Fujii, S., Tanaka, S., Ikebe, N., Beppu, T. et al (1999). Induction of heme oxygenase-1 by nitric oxide and ischaemia in experimental solid tumors and implications for tumor growth. British Journal of Cancer, 80, 1945-1954.

[11] Maines, M.D., Abrahamsson, P.A. (1996). Expression of heme oxygenase-1 (HSP32) in human prostate: normal, hyperplastic, and tumor tissue distribution. Urology, 47, 727-74733.

[12] Hara, E., Takahashi, K., Tominaga, T., Kumabe, T., Kayama, T., Suzuki, H. et al. (1996). Expression of heme oxygenase and inducible nitric oxide synthase mRNA in human brain tumors. Biochemical and Biophysical Research Communications, 224, 153-158.

[13] Goodman, A.I., Choudhury, M., da Silva, J.L., Schwartzman, M.L., Abraham, N.G. (1997). Overexpression of the heme oxygenase gene in renal cell carcinoma. Proceedings of The Society of Experimental Biology and Medicine, 214, 54-61.

[14] Tsutsumishita, Y., Onda, T., Okada, K., Takeda, M., Endou, H., Futaki, S. et al (1998). Involvement of $\mathrm{H}_{2} \mathrm{O}_{2}$ production in cisplatin-induced nephrotoxicity. Biochemical and Biophysical Research Communications, 242(2), 310-312.

[15] Sawa, T., Wu, J., Akaike, T., Maeda, H. (2000). Tumor-targeting chemotherapy by a xanthine oxidase-polymer conjugate that generates oxygen-free radicals in tumor tissue, Cancer Research, $60,666-671$.

[16] Tsutsumi, Y., Kihara, T., Yamamoto, S., Kubo, K., Nakagawa, S., Miyake, M., et al. (1994). Chemical modification of natural human tumor necrosis factor-alpha with polyethylene glycol increases its antitumor potency. Japanese Journal of Cancer Research, 85, 9-12.

[17] Fang, J., Sawa, T., Akaike, T., Maeda, H. (2002).Tumor-targeted delivery of polyethylene glycol-conjugated $\mathrm{D}$-amino acid oxidase for antitumor therapy via enzymatic generation of hydrogen peroxide. Cancer Research, 62(11), 3138-3143.

[18] Matsumura, Y., and Maeda, H. (1986). A new concept for macromolecular therapeutics in cancer chemotherapy: mechanism of tumoritropic accumulation of proteins and the antitumor agent SMANCS. Cancer Research, 46, 6387-6392.

[19] Doi, K., Akaike, T., Fujii, S., Tanaka, S., Ikebe N., Beppu, T. et al. (1999). Induction of heme oxygenase-1 nitric oxide and ischaemia in experimental solid tumors and implications for tumour growth. British Journal of Cancer, 80(12), 1945-1954.

[20] Sahoo, S.K., Sawa, T., Fang, J., Tanaka, S., Miyamoto, Y., Akaike, T. et al. (2002). Pegylated zinc protoporphyrin: a water-soluble heme oxygenase inhibitor with tumor-targeting capacity. Bioconjugate Chemistry, 13(5), 1031-1038.

[21] Fang, J., Sawa, T., Akaike, T., Greish, K., Maeda, H. (2004) Enhancement of chemotherapeutic response of tumor cells by a heme oxygenase inhibitor, pegylated zinc protoporphyrin. International Journal of Cancer. 109(1), 1-8.

[22] El Sayed, S.M., Abou El-Magd, R.M., Shishido, Y., Chung, S.P., Sakai, T., Watanabe, H., et al. (2012). D-amino acid oxidase gene 
therapy sensitizes glioma cells to the antiglycolytic effect of 3bromopyruvate. Cancer Gene Therapy, 19, 1-18.

[23] El Sayed, S.M., Abou El-Magd, R.M., Shishido, Y., Chung, S.P., Diem, T.H., Sakai, T., et al. (2012). 3-Bromopyruvate antagonizes effects of lactate and pyruvate, synergizes with citrate and exerts novel anti-glioma effects. Journal of bioenergetics and biomembranes. $2012 \mathrm{Feb}$; 44(1) :61-79..

[24] Chung, S.P., Sogabe, K., Park, H.K., Song, Y., Ono, K., Abou ElMagd, R.M., et al. (2010). Potential cytotoxic effect of hydroxypyruvate produced from D-serine by astroglial D-amino acid oxidase. Journal of Biochemistry, 148(6), 743-753.

[25] Zhang, W.B., Wang, Z., Shu, F., Jin, Y.H., Liu, H.Y., Wang, Q.J., et al. (2010). Activation of AMP-activated protein kinase by temozolomide contributes to apoptosis in glioblastoma cells via p53 activation and mTORC1 inhibition. The Journal of Biological Chemistry, 285, 40461-40471.

[26] Neman, J., Jandial, R. (2010). Decreasing glioma recurrence through adjuvant cancer stem cell inhibition. Biologics, 4, 157-162.

[27] Colen, C.B., Shen, Y., Ghoddoussi, F., Yu, P., Francis, T.B., Koch, B.J., et al. (2011). Metabolic targeting of lactate efflux by malignant glioma inhibits invasiveness and induces necrosis: an in vivo study, Neoplasia, 13(7), 620-632.

[28] Mulet, C., Lederer, F. (1977). Bromopyruvate as an affinity label for baker's yeast flavocytochrome b2. Kinetic study of the inactivation reaction. European Journal of Biochemistry, 73(2), 443-447.

[29] Fischer, K., Hoffmann, P., Voelkl, S., Meidenbauer, N., Ammer, J., Edinger, M., et al. (2007). Inhibitory effect of tumor cell-derived lactic acid on human T cells. Blood, 109(9), 3812-3819.

[30] Heindel, J.J., Bates, H.K., Price, C.J., Marr, M.C., Myers, C.B., Schwetz, B..A. (1996). Developmental toxicity evaluation of sodium fluoride administered to rats and rabbits in drinking water. Fundamental and Applied Toxicology, 30(2):162-177.

[31] Newby, C.S., Rowland, J.L., Lynch, R.J., Bradshaw, D.J., Whitworth, D., Bosma M.L. (2011). Benefits of a silica-based fluoride toothpaste containing o-cymen-5-ol, zinc chloride and sodium fluoride. International Dental Journal, Suppl 3, 74-80.
[32] Black, M.M., Kleiner, I.S., Bolker, H. (1949). Glycolytic enzyme inhibitor therapy in human malignant neoplasia. Cancer Research, 9(5), 314-319.

[33] Bertrand, R., O'Connor, P.M., Kerrigan, D., Pommier Y. (1992). Sequential administration of camptothecin and etoposide circumvents the antagonistic cytotoxicity of simultaneous drug administration in slowly growing human colon carcinoma HT-29 cells. European Journal of Cancer, 28A(4-5),743-748.

[34] Ko, Y.H., Pedersen, P.L., Geschwind, J.F. (2001). Glucose catabolism in the rabbit VX2 tumor model for liver cancer: characterization and targeting hexokinase. Cancer Letters, 173(1), 83-91.

[35] Ko, Y.H., Smith, B.L., Wang, Y., Pomper, M.G., Rini, D.A., Torbenson, M.S., et al. (2004). Advanced cancers: eradication in all cases using 3-bromopyruvate therapy to deplete ATP. Biochemical and Biophysical Research Communication, 324(1), 269-275.

[36] Ihrlund, L.S., Hernlund, E., Khan, O., Shoshan, M.C. (2008). 3Bromopyruvate as inhibitor of tumour cell energy metabolism and chemopotentiator of platinum drugs. Molecular Oncology, 2(1), 94-101.

[37] Bhardwaj, V., Rizvi, N., Lai, M.B., Lai, J.C., Bhushan, A. (2010). Glycolytic enzyme inhibitors affect pancreatic cancer survival by modulating its signaling and energetics. Anticancer Research, 30(3), 743-749.

[38] Leary, S.C., Hill, B.C., Lyons, C.N., Carlson, C.G., Michaud, D., Kraft, C.S., et al. (2002). Chronic treatment with azide in situ leads to an irreversible loss of cytochrome c oxidase activity via holoenzyme dissociation. The Journal of Biological Chemistry, 277(13), 11321-11328.

[39] Morland, C., Boldingh, K.A., Iversen, E.G., Hassel, B. (2004). Valproate is neuroprotective against malonate toxicity in rat striatum: an association with augmentation of high-affinity glutamate uptake. Journal of Cerebral Blood Flow and Metabolism, $24(11), 1226-1234$ 